\title{
Development of alcohol triggered vehicle engine lock system
}

\author{
Ighalo Joshua ${ }^{1}$, Uzairue Stanley ${ }^{2}$, Ochonogor Charles ${ }^{3}$, Amaize Peter ${ }^{4}$, Kennedy okokpujie ${ }^{5}$ \\ ${ }^{1}$ Department of Electrical \& Electronics Engineering, Federal University Oye-Ekiti, Nigeria \\ ${ }^{2,4,5}$ Department of Electrical and Information Engineering, Covenant University, Nigeria \\ ${ }^{3}$ Department of Electrical and Electronics Engineering, Petroleum Training Institute, Nigeria
}

\section{Article Info}

Article history:

Received Dec 12, 2018

Revised Feb 14, 2019

Accepted Feb 28, 2019

\section{Keywords:}

AT mega 328 PU

microcontroller

DC motor

Drunk drivers

Embedded system

Threshold

\begin{abstract}
Drunk driving is a very dangerous behavior caused as a result of excessive consumption of alcohol therefore causing distortion in the thought pattern of its victims with a large percentage being drivers of vehicles of all forms. Most of the traffic accidents recorded in recent years are related to drunk driving. Solutions have been proposed, devices developed, all to the sole aim which is to reduce traffic accidents due to drunk driving but none has been quite cable of impairing the driver's ability to drive. To this end, we model and design an alcohol triggered vehicle engine lock system. This project's ability to impair the driver's ability to drive makes it stand out from previous methods or devices developed to reduce road accidents due to drunk driving. The entire system is based on a microcontroller that is used to set an alcohol limit/ threshold which when reached or crossed upon sensing of alcohol in the air by the alcohol sensor, would trigger the buzzer alarm and warning LED of the circuit to alert the driver that his/her blood alcohol concentration at that moment in time wouldn't be safe for driving. At this point the system automatically locks the ignition system of the vehicle within which it is embedded while an LCD displays information for the driver's visuals incase the driver's sight isn't also impaired while in the drunk state. This project is a prototype to what is proposed with the vehicle's engine system represented with a DC motor and its ignition system represented with a push button. The overall work was implemented with a constructed work, tested working and perfectly functional.
\end{abstract}

Copyright $\odot 2019$ Institute of Advanced Engineering and Science. All rights reserved.

\section{Corresponding Author:}

Uzairue Stanley,

Department of Electrical and Information Engineering,

Covenant University, Nigeria.

Email: Stanley.uzairue@covenantuniversity.edu.ng

\section{INTRODUCTION}

Drunk driving is a very dangerous behavior caused as a result of excessive consumption of alcohol therefore causing distortion in the thought pattern of its victims. The investigation done by the World Health Organization in 2008 shows that about 50\%-60\% of traffic accidents are related to drunk driving [1]. In present times, the cases of traffic accident caused by drunk driving has increased rapidly. More and more people have realized that drunk driving does great harm to public security. An embedded system consisting of an alcohol sensor tries to reduce the possibilities of accidents caused by drunk driving [1-3]. Embedded systems application in the transportation sector take the form of intelligent transportation systems, intelligent transportation systems include sensing technologies such as infrastructure sensors (in road reflectors) embedded on road sides, automatic number plate recognition technologies, use of gas sensors in detection of dangerous/harmful gases in vehicles etc. all to reduce the increasing rates of accidents of all forms and causes [2]. The alcohol triggered vehicle engine lock system is a prototype device that is developed with the primary purpose of reducing drastically the number of fatal road accidents due to drunk driving by motorist. The system is made up of an alcohol sensor (MQ3 Sensor), AT Mega 328 PU microcontroller, 
Arduino Uno board, DC Motor as model for vehicle engine, push button as model for ignition key of vehicle engine, LCD and buzzer alarm. The system detects alcohol molecules in the air surrounding the consumer of the alcohol via the MQ3 sensor (alcohol sensor) and sends the analog value of the molecules to the AT Mega $328 \mathrm{PU}$ microcontroller which processes the value and compares with the a given threshold which is included in the codes uploaded into the microcontroller. If the value of the alcohol molecules detected is greater than the set threshold, the system automatically prevents the DC motor from operating (i.e. locking the vehicle engine) with the buzzer alarm also going off too and warning state messages popping up on the LCD. But if the value of the alcohol molecules detected is less than the set threshold, the system would allow the DC motor to continue its operation or start up (i.e. not locking the vehicle engine) with the buzzer alarm not going off and the LCD displaying normal state messages.

\section{RELATED WORK}

There have been ideas on the alcohol sensor in recent years starting with simple sensing and detecting circuits by hobbyists to projects channeled towards reducing drastically the number of fatal road accidents due to drunk driving by motorist. One of the works that was initiated towards achieving this goal proposed a device which was made up of the alcohol sensor, AC power supply, LM 358 op amp and LCD circuitry3. When in operation, this system would display the results of the alcohol sensor as it senses the alcohol molecules in air present around it and when it crosses the fixed threshold set by the LM358 op amp. The major merit of this work was that it was the first major headway in the use of the alcohol sensor to drastically reduce the number of fatal road accidents due to drunk driving by motorist and also one of the works that allowed for the availability of alcohol sensor libraries for simulation. The major demerit of this system was inability to the characteristics of portability $[3,4,5]$. It required whosoever to be tested to be close to the AC power outlet due to the system running on AC power unlike the portability that would have been enjoyed if it were powered on DC. Another demerit was the presence of a preset threshold due to the presence of the LM358 op amp in the circuit with any action to respond to the crossing of such threshold. The LM358 op amp which was acting as a comparator in the circuit and came with a preset value [5] for the threshold upon crossed, had no response such as an alarm to warn that the threshold has been crossed. This system was required two participants i.e. one person to carry out the testing and note when the threshold is crossed and the other as the person being tested. This system was nowhere close to proving a means of inhibiting a driver if he/she were drunk not to mention real time implementation.

A recent research proposed an alcohol detection system that alerts the driver through his/her cellphone. This system was a huge advancement from breathalyzers as it was based on GSM technology using the GSM module and dumped the use of an alarm circuit but still employed the LM358 op amp. The system alerted via text messages using a GSM module and had a unique ringtone for such text messages set on the cellphone. This system was also proposed for use in a vehicle for real time implementation which again distinguished positively further from the two previously reviewed works [6]. The system lacked a display unit i.e. LCD due to the presence/use of the cellphone. With all this features highlighted, this system was a huge breakthrough in bridging the gap further in reducing drastically the number of fatal road accidents due to drunk driving by motorist but it had some drawbacks still like another project. Its major demerit was the lack of an LCD unit and an alarm circuit. As generic as the alarm circuit seemed in this work, it is required as since the system was proposed for real time implementation, there would be cases of where the driver would not be with his or her cellphone in the vehicle at the period of drunkenness and as such no means of alerting the driver of his/her state of drunkenness keeping in mind this system doesn't provide any other means of impairing the driver's ability to drive. Another demerit is in the case of the visual and hearing impairment of such driver. If both were to occur at the same time to a particular driver, then there is absolutely no means of alerting the driver.

The presence of the LM358 op amp as a comparator was another drawback as it can be assumed that with the advancement in the technology in use in this system [5], the use of op amp would be ditched and a microcontroller employed to allow for flexibility in changing the blood alcohol concentration (BAC) threshold due to probability in changes of body chemistry of the driver. The issue of cellphone batteries running down also comes up implying that the system would be inactive in the state that a cellphone battery is dead. Also, with most drivers in the habit of keeping their cellphones in the vibration or silent mode while driving, this inhibited the alerting property of the work. Still, this work didn't provide a means of inhibiting the driver's ability to drive just liked the previously reviewed works above provided he/she were in a drunken state. In recent research, a design similar to the previous study was proposed. The major difference between both works was the introduction of a microcontroller in the former's work. Also, this system was to operate on AC power unlike the former which was on the cellphone's battery. The microcontroller used was the PIC 16F877A microcontroller [7]. The microcontroller has a high power consumption rate and required a lot of 
code therefore making it difficult to be recompiled when necessary but this created room for flexibility in the setting of the threshold value and no issue of changing body chemistry of the driver therefore affecting his/her blood alcohol concentration value was supposed to occur $[6,8]$. The presence of the microcontroller, gave room for addition of other features in the future. The only major drawback was the system's inability for a direct real time implementation due to it being powered by an AC power supply, as the alcohol sensor wouldn't have the opportunity to have at least $3 \mathrm{hrs}$ full run in time it would get if on DC supply (vehicle battery) to give the sensor the degree of accuracy it requires for its operation [9]. With all this feature, this work still didn't provide a means of inhibiting the driver's ability to drive just liked the previously reviewed works above provided he/she were in a drunken state. This study is based on the ideas of the previously reviewed works above but with a lot of modification in terms of circuitry all to achieving the goal of inhibiting the driver's ability to start up the vehicle provided the alerting features of the system fails to stop the driver.

\section{METHODS}

\subsection{Component theory}

The Arduino Uno Board is the central unit of the system. The Arduino Uno is a microcontroller board based on the ATmega328 [10]. It is a programmable micro controller for prototyping electromechanical devices. Sensors (Gyroscopes, GPS Locators, accelerometers) \& Actuators (LEDS or electrical motors) just as the MQ3 sensor and DC motor were connected to the board. It has 14 digital Input/output pins (of which 6 can be used as PWM outputs), 6 analog inputs, a $16 \mathrm{MHz}$ ceramic Resonator, a USB connection, a power jack, an ICSP header and a reset button as shown in Figure 1 [11]. It contains everything needed to support the AT Mega 328 PU microcontroller. The Uno board was used to interface the LCD, DC motor, MQ3 sensor, buzzer alarm, push button and Led warning light with the AT Mega 328 PU microcontroller.

The alcohol sensor also known as the MQ3 sensor is suitable for detecting alcohol and was used in this project to detect alcohol as shown in Figure 2 [5]. It has a high sensitivity to alcohol and small sensitivity to Benzene. The sensitivity was adjusted using a $10 \mathrm{~K}$ potentiometer and integrating the presence of the potentiometer in the code to set the threshold. The sensitive material of the MQ-3 gas sensor is $\mathrm{SnO} 2$, which has lower conductivity in clean air. But when the target alcohol gas exists, the sensor's conductivity is higher along with the gas concentration rising [12-14]. LCD (Liquid Crystal Display) screen is an electronic display module and find a wide range of applications as shown in Figure 3. A 16x2 LCD display is very basic module and is very commonly used in various devices and circuits and was used in this project to display both warning and normal messages. A 16x2 LCD means it can display 16 characters per line and there are 2 such lines.



Figure 1. Arduino uno board

In this LCD each character is displayed in 5x7 pixel matrix. The LCD was connected to the Arduino Uno board via the ports on the board so as to interface it with the microcontroller and allow for programming of the LCD [15]. This DC or direct current motor works on the principle, when a current carrying conductor is placed in a magnetic field, it experiences a torque and has a tendency to move as shown in Figure 4 . This is known as motoring action. If the direction of current in the wire is reversed, the direction of rotation also reverses. When magnetic field and electric field interact, they produce a mechanical force, and based on that 
the working principle of dc motor established. The direction of rotation of this motor is given by Fleming's left hand rule, which states that if the index finger, middle finger and thumb of your left hand are extended mutually perpendicular to each other and if the index finger represents the direction of magnetic field, middle finger indicates the direction of current, then the thumb represents the direction in which force is experienced by the shaft of the dc motor. In this project, the DC motor was used as a prototype for the vehicle engine [16].

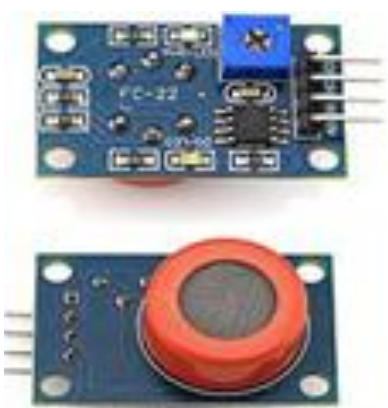

Figure 2. MQ3 gas sensor

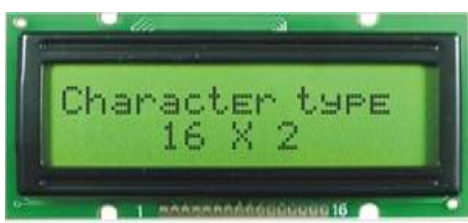

Figure 3. LCD

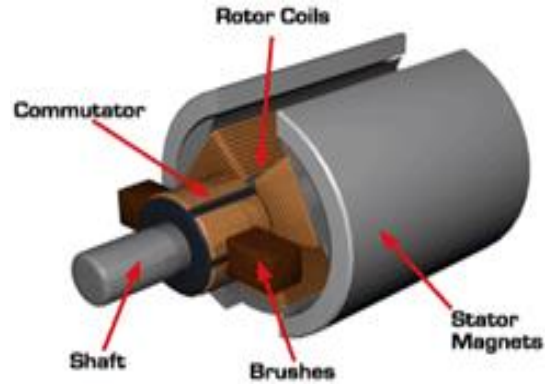

Figure 4. DC motor

\subsection{Principle of Operation}

This section presents the design of the software and hardware part of the project. Proteus and Arduino were the main software used during this project. Simulation in proteus was done to study the behavior of the system before the hardware design. Block diagram of the system as shown in Figure 5.

With the aid of Figures 5-6 the mode of operation of this project is described. Whenever the ignition of the engine is started, the sensor measures the content of the alcohol in the breath of the driver or in the air around the sensor close to the driver and automatically switches off the engine if the driver is found to be drunk. In this system the sensor delivers a current with a linear relationship to the alcohol molecules from zero to very high concentration. The output of the sensor is fed to the ATmega 328pu for comparison. If the measured value reaches or exceeds the threshold, the motor stops automatically and the buzzer produces sound with the LCD displaying warning messages and a warning LED coming up. But if the measured value is less than the threshold, the motor can still start up or continue running if already started with no buzzer alarm going off, LCD displaying "normal" message and no warning LED glowing. The project's operational flowchart in Figure 6 describes the mode of operation of the system.
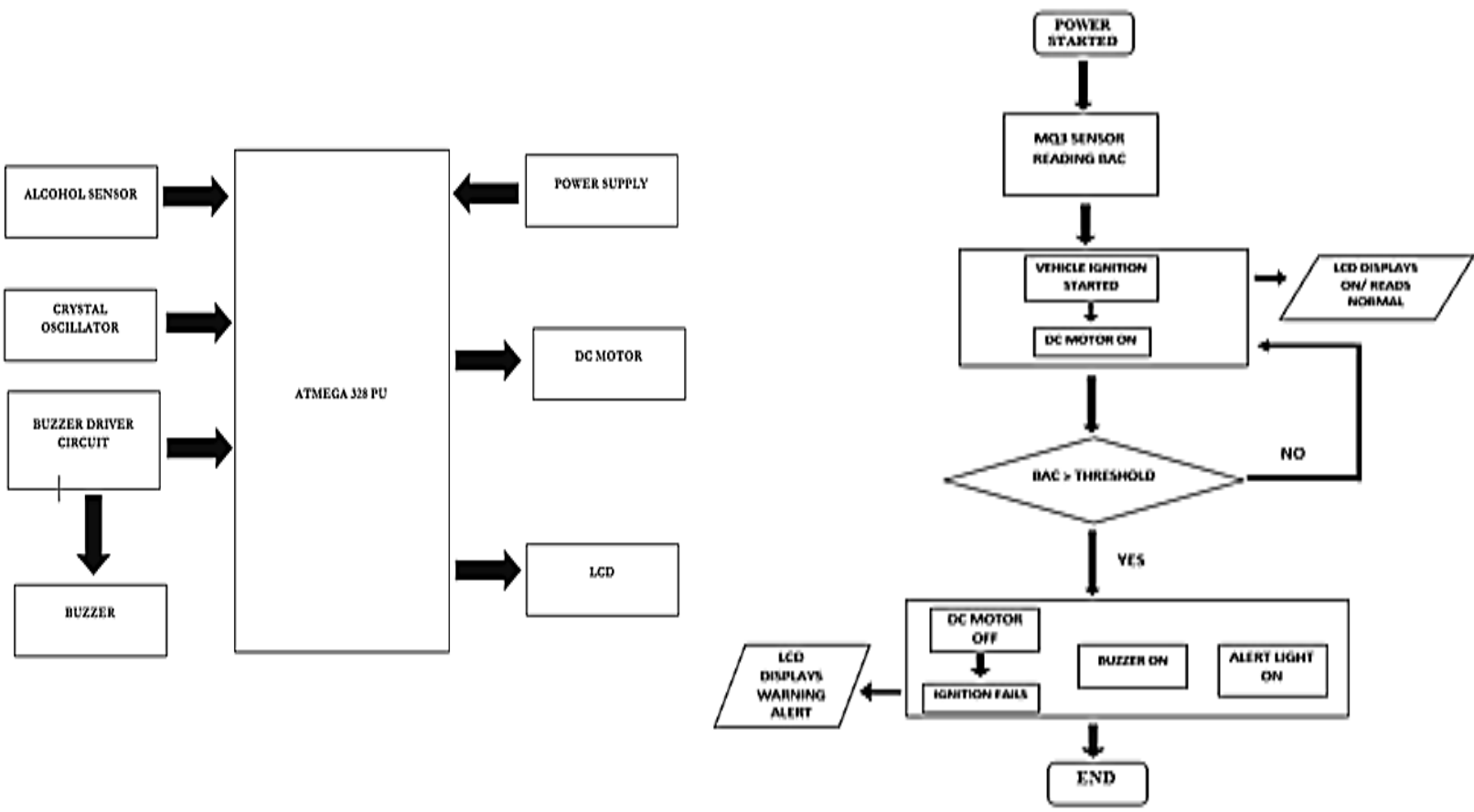

Figure 5. Block diagram of the system

Figure 6. The project operational flow chart 


\subsection{Power supply unit}

The power supply unit consists of a voltage regulator (7805), $12 \mathrm{~V}$ battery, filter capacitors and a resistor as shown in Figure 7. The voltage source for this system was a 12V DC battery. This was chosen based on the range of values that the voltage regulator used for the project requires which is from $7 \mathrm{~V}-20 \mathrm{~V}$. The two capacitors used in this stage of the project are two electrolytic capacitors as they gave the power supply section low leakage resistance feature (at input and output of the voltage regulator respectively) and were selected instead of the conventional and (input and output of the voltage regulator respectively) stated in the LM7805 datasheet because the whole the system will pull power from power source for some time. The voltage regulator used in this project was the LM7805 voltage regulator.

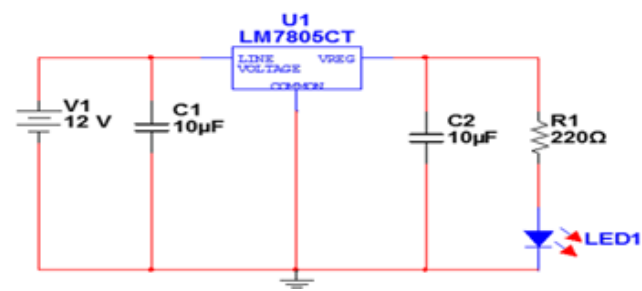

Figure 7. Power supply circuit

This type of voltage regulator was selected because its output of $5 \mathrm{~V}$ is required for operation by the microcontroller, MQ3 sensor, LCD and DC motor. As seen in the circuit below, the first capacitor, the electrolytic capacitor, was hooked up after the voltage source, in this case the $12 \mathrm{~V}$ battery and before the input of the LM7805 regulator. This capacitor filters out any noise coming from the battery. The capacitor, in essence, acts as a bypass capacitor. It shorts the AC signal of the voltage signal (which is noise on the voltage signal) to ground and only the DC portion of the signal goes into the regulator. The second capacitor, the electrolytic capacitor, is hooked up after the voltage regulator. This capacitor is there again to filter out any noise or high-frequency (ac) signals that may be on the DC voltage line therefore outputting a precise voltage i.e. $5 \mathrm{~V}$ required to power the microcontroller.

\subsection{Sensing Stage}

\subsubsection{MQ3 Sensor}

The MQ3 sensor in combination with the LM393 was used in this project for detecting alcohol and processing the detected alcohol into signals that is interpreted by the microcontroller. Figure 8 shows a full circuit diagram of the sensor.

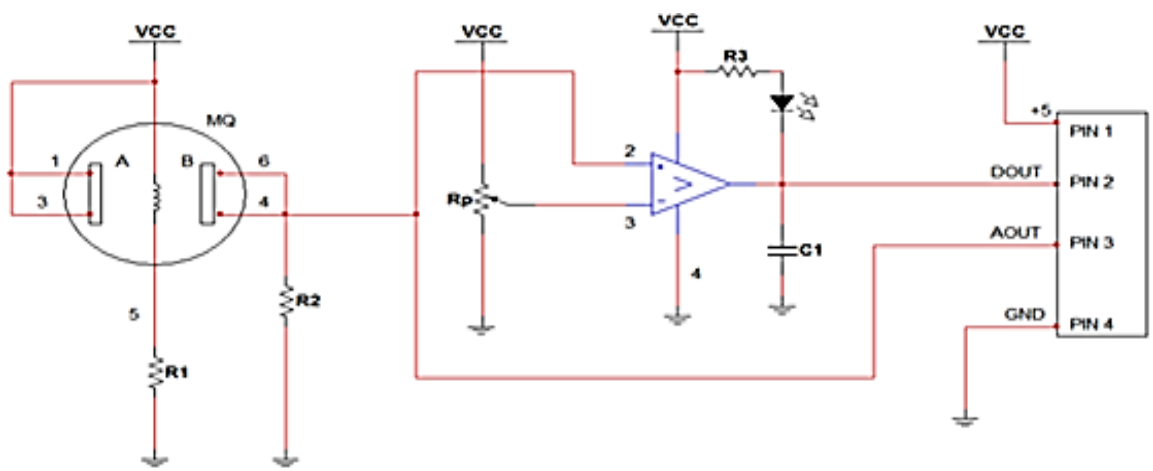

Figure 8. Circuit diagram of MQ3 Sensor

The MQ3 module itself has four pins. It has an analog output pin, and a digital output that is supplied from a comparator output. The digital output is normally high. When alcohol molecules become present in the surroundings of the sensor, the sensor output swings from $5 \mathrm{v}(1)$ to $0 \mathrm{v}(0)$. This output was further calibrated in the project with the aid of a $10 \mathrm{~K}$ variable resistor Figure 9 is a schematic diagram of the MQ3 element alone. 


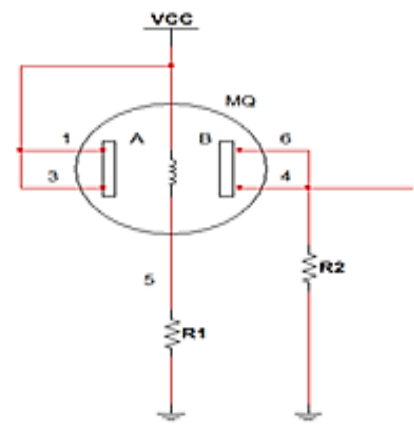

Figure 9. Schematic of the MQ3 element

The resistances across the A pin and B pin varies depending on how much alcohol is in the air around the sensor. Alcohol in the breath is represented in resistance. The more alcohol, the lower the resistance, instead of measuring the resistance directly, we measure the voltage level at the point between the sensor and a load resistor R2The sensor and load resistor R2 form a voltage divider, and the lower the sensor resistance, the higher the voltage reading which was then fed directly into the input of the LM393 comparator.

\subsubsection{Sensing Stage; Comparator Unit}

The LM393 is the comparator circuit used and it compared two voltage signals and determined which one was greater. The result of comparison is indicated by the output voltage. The comparator functions in the following manner. It gives a high output when the voltage in the non-inverting input (+) is greater than the voltage at the inverting input (-) and it gives a low output when the voltage at the inverting input (-) is greater than or equal to the voltage in the non-inverting input $(+)$. Figure 10 is a circuit diagram of the LM393 comparator.

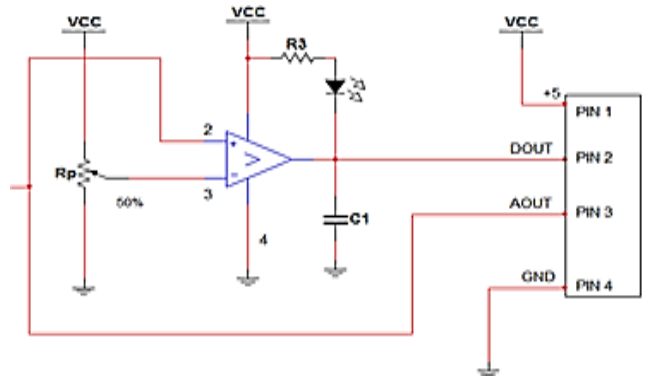

Figure 10. Circuit diagram of LM393 voltage comparator

In the comparator stage a precise point is set where the output voltage changes. This is achieved by adjusting voltage of $R_{P}\left({ }_{R_{F}}\right)$ in Figure 10 above. Setting $V_{R_{F}}$ to $2.0 \mathrm{~V}$ implies that any voltage below $2.0 \mathrm{~V}$ in the inverting input (when alcohol molecules are present in the surrounding air of the sensor module) will make the comparator output HIGH. Any voltage above $2.0 \mathrm{~V}$ in the inverting input will make the comparator output LOW. The comparator would therefore give a HIGH when there were alcohol molecules present in the air surrounding the sensor module and a LOW when there were no alcohol molecule present in the air surrounding the sensor module. This shows how sensitive the sensor is (i.e. the sensor would immediately sense the presence of alcohol molecules no matter the quantity present) and therefore a $10 \mathrm{~K}$ potentiometer was added to enable calibration of the sensor via the microcontroller. The output of the comparator is sent to the microcontroller for interpretation and actions to be taken via the AOUT and DOUT pin.

\subsection{Calibration of the MQ3 Sensor Module}

Set threshold $=244$, AOUT value range $=0-1024$

$\%$ alcohol in breath $=\frac{244}{1024} \times 100=23.83 \% \approx 0.2383$ 
$\operatorname{BAC}(\mathrm{mg} / \mathrm{L})=($ alcohol in breath $) \times\left(\frac{2100}{(10000}\right)$

$\operatorname{BAC}(\mathrm{mg} / \mathrm{L})=(0.2383) \times\left(\frac{2100}{10000}\right)=0.05 \mathrm{~g} / 100 \mathrm{~mL}=0.5 \mathrm{~g} / \mathrm{L}$

The calculation verifies that the set threshold of 244 gives a BAC value of $0.5 \mathrm{~g} / \mathrm{L}$ as required for the project. The MQ3 detects alcohol molecules which are measured as analog reading (sensor value). A $10 \mathrm{~K}$ potentiometer is connected to the analog pin (A1) of the microcontroller that aids in the calibration by holding the threshold analog value which when crossed makes the system declare the driver drunk. The following terms below are included into the program for the project so as enable successful calibration of the sensor. The following terms below are included into the program for the project so as enable successful calibration of the sensor.

const int analogPin $=\mathrm{A} 1$;

const int threshold $=244$;

void $\operatorname{loop}()\{$

int analogValue $=$ analogRead(AOUTpin);

if (analogValue $>$ threshold) \{\}

else \{\}

\subsection{Display Unit}

The display used in this project is the liquid crystal display (LCD). It displays information of the project based on what information it has being programmed using the microcontroller to display. The $16 \mathrm{X} 2$ LCD is employed in the display unit as it is economical; easily programmable; has no limitation of displaying special \& even custom characters (unlike in seven segments). The LCD is connected according to the guidelines as directed in the datasheet but a $10 \mathrm{~K}$ potentiometer and a $220 \Omega$ resistor is connected to the LCD so as tune the LCD for better clarity of characters and reduce the brightness of the display respectively. Circuit diagram of alcohol triggered vehicle engine lock system as shown in Figure 11.

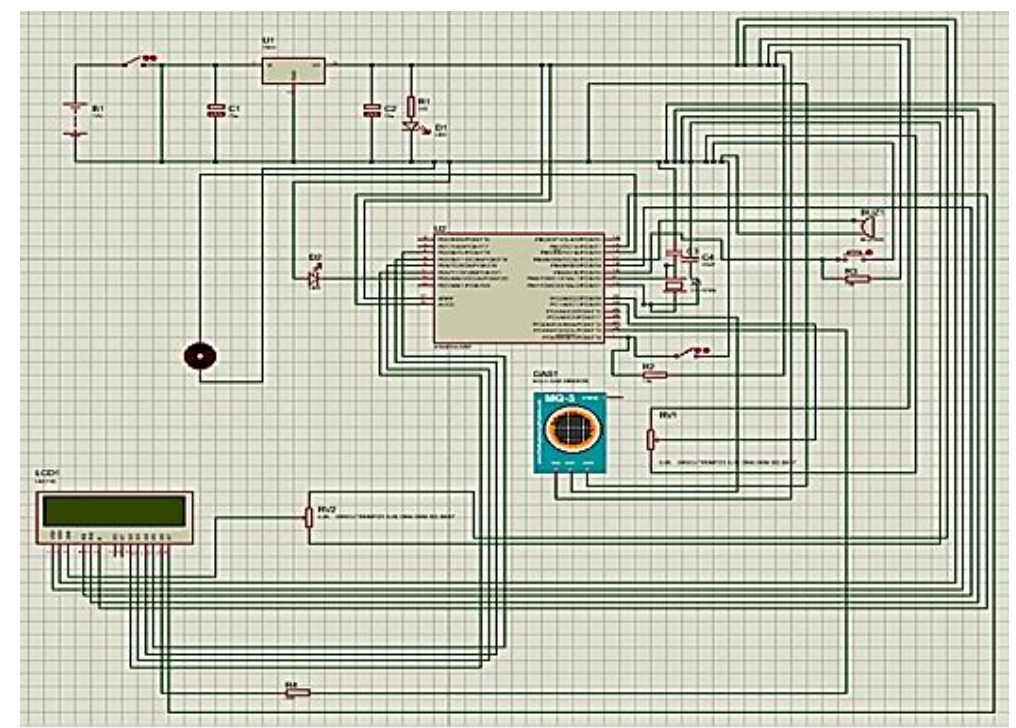

Figure 11. Circuit diagram of alcohol triggered vehicle engine lock system

\section{RESULTS}

The Table 1 shows the results taken from placing a specific volume of alcohol at specific distances from the MQ3 sensor as highlighted in table below. The analog value of the blood alcohol concentration at each distance is also gotten. The set threshold for the project was 244 (analog value) i.e. $0.5 \mathrm{~g} / \mathrm{l}$ (BAC value). Figure 12 is a graph of the blood alcohol concentration against the distance between the sensor and the volume of alcohol. It is deduced from the graph that as the distance increases the BAC and analog value decreases i.e. Distance between the sensor and the volume of alcohol is inversely proportional to blood alcohol concentration and the analog value. 


\begin{tabular}{|c|c|c|}
\hline Distance $(\mathrm{cm})$ & Analog value & BAC $(g / l)$ \\
\hline 0 & 320 & 0.66 \\
\hline 10 & 260 & 0.53 \\
\hline 20 & 155 & 0.32 \\
\hline 30 & 126 & 0.26 \\
\hline 40 & 94 & 0.19 \\
\hline 50 & 87 & 0.18 \\
\hline
\end{tabular}

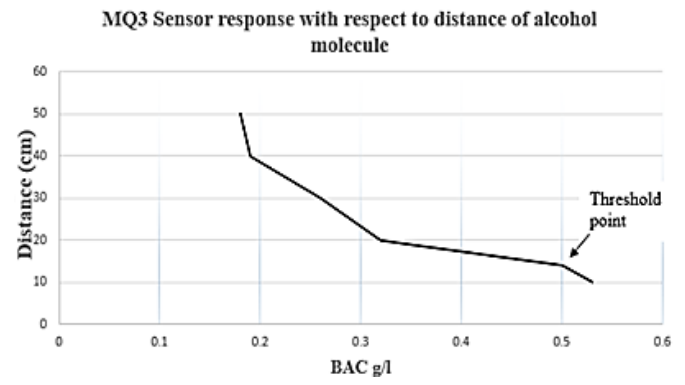

Figure 12. Graph of Blood alcohol concentration vs Distance between sensor and alcohol $(\mathrm{cm})$

\section{DISCUSSIONS}

Several problems were encountered during the project. The problems range from design problems to implementation problems and also construction problems. The major problems are as follows. The MQ3 sensor cannot differentiate between alcohol molecules and perfumes due to the presence of ethanol in both as the sensor reacts to any form of matter with high concentration of ethanol and as such a driver who isn't drunk and has used a lot of perfume could trigger the system. The $9 \mathrm{~V}$ battery was not supplying enough current to power the circuit and the sensor at the same time. This problem was solved by using a $12 \mathrm{Volts}$ battery instead of a 9Volts battery. The buzzer was not loud enough when connected to the microcontroller alone. This problem was solved by connecting the buzzer to a transistor so as to amplify it as the microcontroller port doesn't have enough current to power the buzzer to operate at its normal capacity. The voltage regulator got was getting very hot due to the high amount of power dissipating as heat and as such leading to the voltage regulator switching off automatically. This problem was solved by attaching a copper heat sink to the voltage regulator. When the whole system was switched on, the LCD was not displaying any characters. This problem was solved by connecting a $10 \mathrm{~K}$ potentiometer to the LCD as it was used to tune the contrast of the LCD. Other problems include soldering and measurement errors but these problems were solved by proper troubleshooting with serious care in the construction of the project.

\section{CONCLUSION}

The project which is the design and implementation of an alcohol triggered vehicle engine lock system has met its objectives. The project is a real time implementation of the prototype of the model and was done considering some factors such as economy, availability of components and research materials, efficiency, compatibility, portability and also durability. The general operation and performance of the project is dependent on the presence of alcohol within $10 \mathrm{~cm}$ range of the system which consist of the alcohol sensor. If the quantity of alcohol detected by the system is greater than the threshold set in the system, the system's buzzer alarm is triggered while its warning LEDs come up and a warning message is displayed by the LCD. But if the quantity of alcohol detected by the system is less than the set threshold in the system, the system's buzzer stays low while its warning LEDs does not come on with a normal message instead of a warning message displayed by the LCD. The operation of the system is also dependent on how well the soldering was done, and the positioning of the components on the Vero board in which proper soldering was done and the components were well spaced on the Vero board with the IC's were soldered away from the power supply stage to prevent heat radiation which, might occur and affect the performance of the entire system. Construction of the system was done in such a way that it makes maintenance and repairs an easy task and affordable for the user should there be any system breakdown with troubleshooting made easier due to soldering of the whole project on just one Vero board. The design of the microcontroller based automatic vehicle engine lock system for drunken drivers involved research in both analog $\&$ digital electronics with intensive work done on microcontrollers and programming in C. In general, the system was designed, and the real time implementation was done with a proto-type of the model.

\section{FUTURE WORKS}

There are no projects that cannot be improved. Enhancements have to be carried out so as to improve the efficiency of this system. One of the improvements that could be made on this system in the future is that it should be made smaller. The smaller the system, the more convenient the alcohol system is, the more likely drivers will accept it. 
There should be proper positioning of the alcohol sensor so as to allow convenient reading of the driver's alcohol consumption quantity with or without the aid of the driver. Some options for where the sensor can be placed include;

- An element that can help differentiate the ethanol quantity in alcohol and perfumes should be introduced into the sensor so as to prevent the issue of the system being triggered due to use of large amount of perfume by the driver.

- A cable can be put near the driver's seat and then connected with the ignition of the car. This means the alcohol detection system can be another key to the car. The driver should blow to the system before he/she start the car. If the value of the alcohol concentration is above the system's threshold value, the system will stop the car starting. So a drunk driver would not be able to start the car which will prevent the behavior of drunk driving. It is not only safe to the driver, but can ensure the passengers would not be hit because of the driver's drunk driving.

\section{REFERENCES}

[1] Killoran, A., Canning, U., Doyle, N., \& Sheppard, L., "Review of effectiveness of laws limiting blood alcohol concentration levels to reduce alcohol-related road injuries and deaths," Final Report, London: Centre for Public Health Excellence (NICE), 2010.

[2] Embedded system. http://en.wikipedia.org/wiki/embedded_system (accessed July 2017).

[3] Lee, K. (2010). "Assessing the Feasibility of Vehicle-Based Sensors to Detect Alcohol Impairment.," National Highway Traffic Safety Administration: Washington, DC. 2010.

[4] RTOS Kernel. http://rtos.com/ (accessed May 2017).

[5] MQ3 Sensor Datasheet. http://www.sparkfun.com/datasheets/sensors/MQ-3.pdf (accessed may 2017).

[6] Kong, H., Rue, X., \& Dai, J. (2013). Alcohol detecting system (Project report).

[7] Phani, S.A., Samuel, S.V., Kaylan, G.C., \& Ravi, T.G. (2014). Liquor detection through automatic motor locking system: In built (LDAMLS). International Journal of Computational Engineering Research (IJCER), Vol 4, Issue 7, July-2014.

[8] Brain, M. Microcontroller.http://electronics.howstuffworks.com/microcontroller.html/ (accessed July 2017).

[9] James, N., Aparna C., \& Teena P. (2014). Alcohol detection system. International Journal of Research in Computer and Communication Technology, Vol 3, Issue 1, January- 2014.

[10] Microcontroller \& Microprocessor. http://engineersgarage.com/tutorials/difference-between-microprocessor-andmicrocontroller/ (accessed July 2017)

[11] Atmel 42735B $\quad$ ATmega 432 Datasheet. http://newbiehack.com/microcontrollerIntroductionABeginnersGuideToTheAtmelAVRAtmega32series. (accessed July 2017).

[12] MQ Gas Sensors. http://playground.arduino.cc/Main/MQGasSensors. (accessed July 2017).

[13] Sensor Report - MQ3 Gas sensor. http://sensorworkshop.blogspot.com/2008/04/sensor-report-mq3-gas-sensor.html/ (accessed July 2017).

[14] Fraden, J. (2010). Handbook of modern sensors: Physics, designs, and applications: Springer, 2010.

[15] LCD Display. http://www.sparkfun.com>datasheets (accessed July 2017).

[16] DC Motor. http://www.electrical4u.com/dc-motor-or-direct-current-motor/, (accessed July 2017). 